\title{
Deciphering the role of interferon alpha signaling and microenvironment crosstalk in inflammatory breast cancer
}

\author{
Olivia K. Provance ${ }^{1}$ and Joan Lewis-Wambi ${ }^{1,2^{*}}$
}

\begin{abstract}
Inflammatory breast cancer (IBC) is the most rare and aggressive subtype of breast cancer characterized by clusters of tumor cells invading lymph vessels, high rates of metastasis, and resistance to systemic chemotherapy. While significant progress has been made in understanding IBC, survival among IBC patients is still only one half that among patients with non-IBC. A major limitation to the development of more specific and effective treatments for IBC is a lack of identifiable molecular alterations that are specific to IBC. Emerging evidence suggests that the aggressive nature of IBC is not specific to IBC cells but instead driven by the interplay between autonomous signaling and context-dependent cytokine networks from the surrounding tumor microenvironment. Recently, the type I interferon, specifically the interferon alpha signature, has been identified as a pathway upregulated in IBC but few studies have addressed its role. Activation of the interferon alpha signaling pathway has been shown to contribute to apoptosis and cellular senescence but is also attributed to increased migration and drug resistance depending on the interferon-stimulated genes transcribed. The mechanisms promoting the increase in interferon alpha expression and the role interferon alpha plays in IBC remain speculative. Current hypotheses suggest that immune and stromal cells in the local tumor microenvironment contribute to the interferon alpha signaling cascade within the tumor cell and that this activation may further alter the immune and stromal cells within the microenvironment. This review serves as an overview of the role of interferon alpha signaling in IBC. Ideally, future experiments should investigate the mechanistic interplay of interferons in IBC to develop more efficacious treatment strategies for IBC patients.
\end{abstract}

Keywords: Inflammatory breast cancer, Interferon alpha, Interferon-stimulated genes, IFITM1, STAT, Dendritic cells, Macrophages, Fibroblasts, Endothelial cells

\section{Background}

Inflammatory breast cancer (IBC) is the most aggressive and lethal subtype of breast cancer accounting for $3 \%$ of total breast cancer diagnoses but $10 \%$ of total breast cancer-related deaths [1]. As of 2018, the 5-year survival rate for IBC is $40-60 \%$ [2] and the 15-year survival rate is approximately $20 \%$ [3]. IBC presents with distinctive features that are absent in non-IBC allowing for a clinical diagnosis. These features include rapid redness and swelling of the breast, generalized induration, and peau d'orange skin consistency often accompanied by a pathological

\footnotetext{
* Correspondence: jlewis-wambi@kumc.edu

'Department of Cancer Biology, University of Kansas Medical Center, 3901 Rainbow Boulevard, Wahl Hall East 1031, Kansas City, KS 66160, USA

${ }^{2}$ The University of Kansas Cancer Center, 3901 Rainbow Boulevard, Kansas City, KS 66160, USA
}

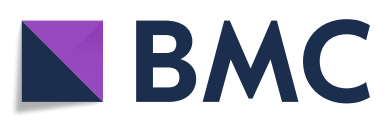

(c) The Author(s). 2019 Open Access This article is distributed under the terms of the Creative Commons Attribution 4.0 International License (http://creativecommons.org/licenses/by/4.0/), which permits unrestricted use, distribution, and reproduction in any medium, provided you give appropriate credit to the original author(s) and the source, provide a link to the Creative Commons license, and indicate if changes were made. The Creative Commons Public Domain Dedication waiver (http://creativecommons.org/publicdomain/zero/1.0/) applies to the data made available in this article, unless otherwise stated.

confirmation of invasive carcinoma through a skin-punch biopsy [2]. It is hypothesized that the clinical symptoms manifest due to blockage of dermal lymph vessels by aggregates of tumor, immune, and stromal cells termed tumor emboli, a hallmark of IBC [2]. Despite these differences, IBC is similar to non-IBC in terms of molecular subtyping. All breast tumors are characterized based on receptor status (estrogen receptor, ER; progesterone receptor, PR; human epidermal growth factor-2, HER2) and a panel of genes contributing to predictive and prognostic indices [4]. IBC is comprised of the same molecular subtypes as non-IBC: ER/PR+ (35-40\%, 60-70\% in non-IBC), HER2+ $(35-50 \%, 20 \%$ in non-IBC) and triple-negative (TN) $(20$ $40 \%, 15-20 \%$ in non-IBC) [5-7]. Though the molecular subtypes overlap, to date, IBC remains significantly more aggressive and there is no IBC-specific therapy. The current 
treatment involves a trimodality approach of neoadjuvant chemotherapy, mastectomy, and axillary lymph node removal followed by post mastectomy radiation [2]. Despite aggressive treatment, IBC patients face significantly higher incidence of metastatic disease compared to non-IBC; therefore, there is an unmet need to determine efficacious treatment strategies for IBC.

Researchers have turned to genetic profiling to identify molecular alterations distinct to IBC; however, these studies remain inconclusive perhaps due to the additional complexity driven by the tumor microenvironment (TME) [8]. Key components of the TME in IBC include dendritic cells, macrophages, fibroblasts, and endothelial cells. The presence of these cells within the microenvironment may contribute to the intrinsic factors of IBC. For example, cancer-associated fibroblasts are a key driver of breast cancer progression [9], macrophages mediate IBC migration $[10,11]$, and IBC tumors have a high infiltration of endothelial cells secreting angiogenic factors such as vascular endothelial growth factor (VEGF) and platelet-derived growth factor (PDGF) $[8,12]$. Though studies have begun to elucidate the tumor-promoting interplay of these cell types, much research is still needed.

Recently, studies have suggested that IBC cells have increased levels of interferon alpha (IFN $\alpha)$ [13, 14]. Interferons are cytokines known for their ability to modulate the innate and adaptive immune system through both autocrine and paracrine signaling [15], providing an interesting and novel avenue for IBC microenvironment research. While direct analysis supporting a role for IFN $\alpha$ signaling in IBC is sparse, extrapolation of data from aggressive breast tumors provides insight into the role of IFN $\alpha$ in IBC. In this review, we summarize the pre-clinical findings supporting a role for IFN $\alpha$ in IBC progression, discuss the potential molecular mechanisms regulated by IFN $\alpha$ in IBC, and predict how the signaling interplay between the microenvironment and IFN $\alpha$ secreted by IBC tumor cells contribute to the pro-tumorigenic milieu. Though we recognize that IFN $\beta$ and IFN $\gamma$ also play an important role in breast cancer progression, our review will focus solely on IFN $\alpha$.

\section{Interferon alpha in inflammatory breast cancer}

In 2014, Bertucci et al. [13] published a study with the largest cohort of non-treated IBC samples to date. DNA microarray expression profiling identified the IFN $\alpha$ pathway as being the only pathway that was significantly upregulated in IBC. Pre-clinical evidence supports this finding but suggests that IFN $\alpha$ upregulation is specific to TN-IBC [14]. In comparing the SUM149 and SUM190 cell lines (TN-IBC and HER2+ IBC, respectively), the mRNA and the secreted levels of IFN $\alpha$ are significantly higher in TN-IBC compared to HER2+ IBC [14]. Though interferons are known to confer an antiviral state to cells, evidence suggests that breast cancer tumors expressing high IFN response genes are 1.7 times more likely to metastasize as compared to tumors expressing low levels of these genes, providing pre-clinical evidence for a pro-tumor face of IFN $[16]$.

\section{An overview of interferon alpha biology and signaling}

IFN $\alpha$ belongs to the family of type I interferons which also consists of IFN $\beta$ [17]. Thirteen subtypes of IFN $\alpha$ have been identified. Each member of IFN $\alpha$ lacks distinct introns and is encoded independently with genes located in a 400-kb cluster on chromosome 9q [18]. Notably, IFN $\alpha$ and IFN $\beta$ both signal through the interferon alpha receptor-(IFNAR) 1 and IFNAR2 complex but have different binding affinities thus producing distinct antiviral effects [17]. Therefore, IFN $\alpha$ and IFN $\beta$ are not interchangeable.

For interferons to elicit their effect, they must bind to their specific receptor which is outlined in Fig. 1a (Adapted from [19]). IFN $\alpha$ binds to IFNAR1 stimulating a conformational change and recruitment of IFNAR2 to dimerize. IFNAR1 and IFNAR2 do not have intrinsic kinase activity but are constitutively associated with Janus-activated kinase-1 (JAK1) and tyrosine kinase-2 (TYK2) [20]. Once IFN $\alpha$ binds to IFNAR1, a conformational change occurs and IFNAR2 dimerizes bringing JAK1 and TYK2 into close proximity with one another promoting cross phosphorylation of JAK1 and TYK2 and subsequent phosphorylation of the intracellular domain of IFNAR1 and IFNAR2 [20]. Phosphorylation of the IFNAR receptors provides a receptor-docking site for signal transducers and activators of transcription (STAT)-1 and STAT2. STAT1 and STAT2 are recruited to the receptors through their $\mathrm{SH} 2$ domain thus allowing them to be phosphorylated by JAK1 and TYK2. Phosphorylated STATs may then homodimerize or heterodimerize. Homodimers of STAT1 are known as IFN $\alpha$-activated factor (AAF) whereas heterodimers of STAT1/STAT2 associated with interferon regulatory factor (IRF)-9 is termed interferon-stimulated gene factor-3 (ISGF3). These complexes translocate into the nucleus and bind to gamma-activated sequences (GAS) or interferon-stimulated response elements (ISREs) in DNA, respectively, transcribing interferon-stimulated genes (ISGs) which are listed in Table 1 (adapted from [21-23]).

Gene transcription mediated by IFN $\alpha$ is tightly regulated by multiple processes including stability of the IFN $\alpha /$ IFNAR1/IFNAR2 complex, the concentration of IFNAR $1 / 2$ on the membrane and transcription of negative regulators including suppressor of cytokine signaling (SOCS) and ubiquitin-specific peptidase (USP) 18 [17]. An in-depth discussion of these regulatory processes and how it pertains to IBC is beyond the scope of this review but has been previously discussed [17]. Regardless, these factors are important to consider for future studies on IFN $\alpha$ signaling in IBC. 

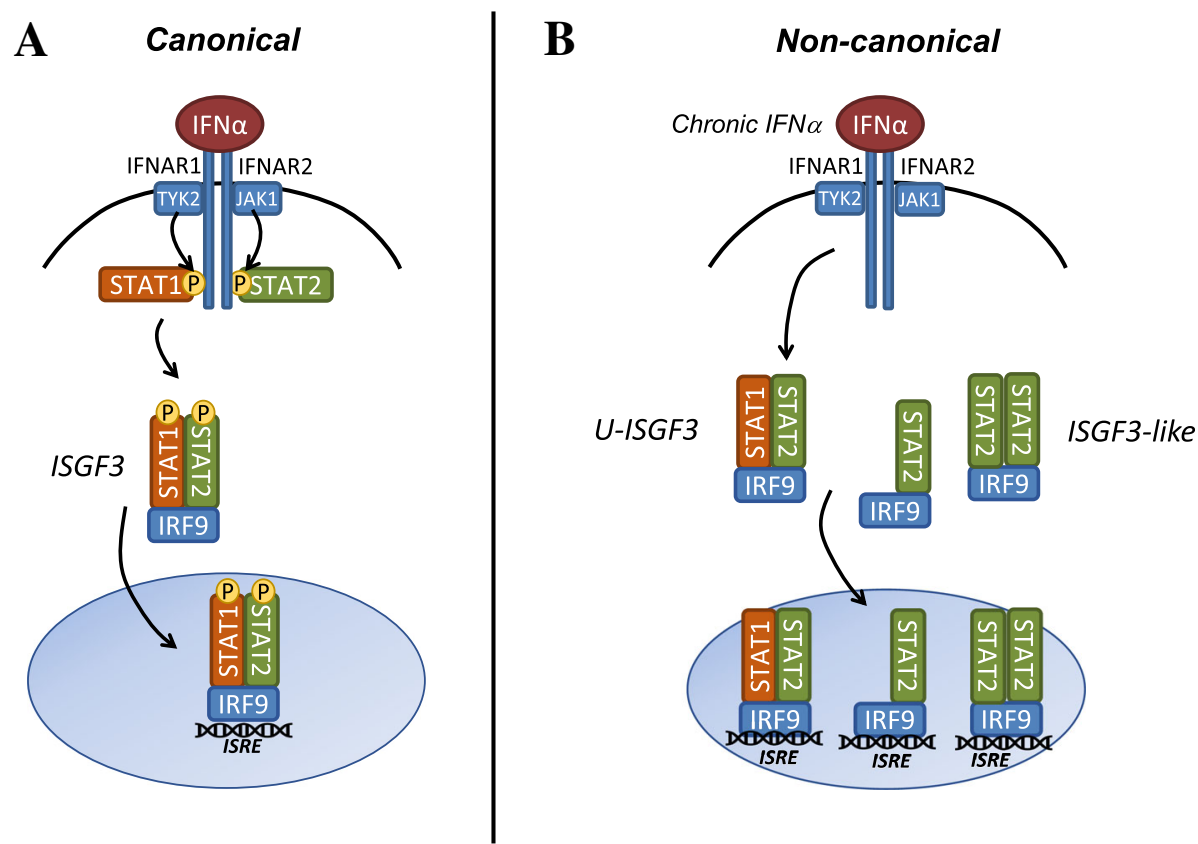

Fig. 1 Downstream IFNa signaling in IBC. a Canonical IFNa signaling begins with IFNa binding to IFNAR1/IFNAR2 receptor. After it binds to the receptor, JAK1 and TYK2 cross-phosphorylate each other and phosphorylate the intracellular domains of the receptors to allow for STAT1 and STAT2 binding through their SH2 domain for subsequent phosphorylation. Once STAT dimers form, they translocate into the nucleus and bind to their respective DNA binding elements. IRF9 and STAT1 are the only two proteins that directly interact with the DNA whereas STAT2 is necessary for stabilizing the ISGF3 complex and further recruitment of co-activators. b During chronic IFNa signaling, STAT proteins are no longer robustly phosphorylated however the interferon signature may remain upregulated due to the similarity between ISGF3 and U-ISGF3. In the absence of IFNa, there will either be no signal or, depending on STAT2 and IRF9 levels, the interferon response may stay elevated due to the continued formation of STAT2 and IRF9. TN-IBC cells have robust levels of IRF9 and IRF9 increases in tumor clusters. Therefore, IRF9 could be the key driver of this response. Adapted from [19]

\section{Intracellular regulation of interferon alpha production Transcriptional regulation of interferon alpha}

Stimulation of IFN $\alpha$ transcription is a complex process and is outlined in Fig. 2. Canonically, production of IFN $\alpha$ is driven by activation of endosomal membrane localized toll-like receptors (TLRs) or cytoplasmic localized retinoic acid-inducible gene I (RIG1) receptors

Table 1 Transcription factor complexes and their control of interferon-stimulated genes

\begin{tabular}{llll}
\hline ISGF3 & & U-ISGF3 & GAS \\
\hline MX1/2 & IRF9 & MX1/2 & IFITM1 \\
IFITM1 & IRF4 & IFITM1 & STAT4 \\
PLSCR1 & CGAS & PLSCR1 & IRF1 \\
OAS1/2/3 & STAT1 & OAS1/2/3 & IRF8 \\
SOCS & STAT2 & IRF7 & IDO \\
USP18 & NOS2 & STAT1 & NOS2 \\
ISG15 & & IFI27 & PDGFRA \\
IRF3 & & IFI44 & KRT14 \\
IRF7 & & & IRF2 \\
\hline ISGF3 (inf
\end{tabular}

ISGF3 (interferon-stimulated gene factor 3), consists of phosphorylated STAT1 and STAT2 dimers bound to IRF9; U-ISGF3 (unphosphorylated interferonstimulated gene factor 3), consists of a dimer of unphosphorylated STAT proteins bound to IRF9; GAS (gamma activated sequence), consists of phosphorylated STAT1 dimers and cyclic GMP-AMP synthase (cGAS) receptor by combinations of double-stranded DNA (dsDNA), double-stranded RNA (dsRNA), single-stranded RNA (ssRNA), or by viral DNA [24, 25]. Adaptor molecules associated with the receptors then stimulate specific kinase activity promoting phosphorylation of interferon regulatory factor (IRF3) and IRF7 or degradation of IkB for $N F \kappa B$ translocation into the nucleus [22]. Importantly, each IFN $\alpha$ family member is transcribed by IRF3 or IRF7; however, the binding sites for these transcription factors are slightly altered in each gene [26]. Since the majority of what is known about IFN $\alpha$ transcription is derived from studies focusing on viral replication, we will discuss the potential regulatory mechanisms promoting increased IFN $\alpha$ levels in IBC through extrapolating data from viral activation and breast cancer in general and applying it to IBC.

\section{Mechanisms contributing to interferon alpha transcription in IBC}

In the absence of bacterial or pathogenic signals, cancer cells can transcribe IFN $\alpha$ in response to endogenous danger signals driven by persistent DNA damage response because of chromosomal instability (CIN) [27] (Fig. 2). Cancer cells have a high error rate of 


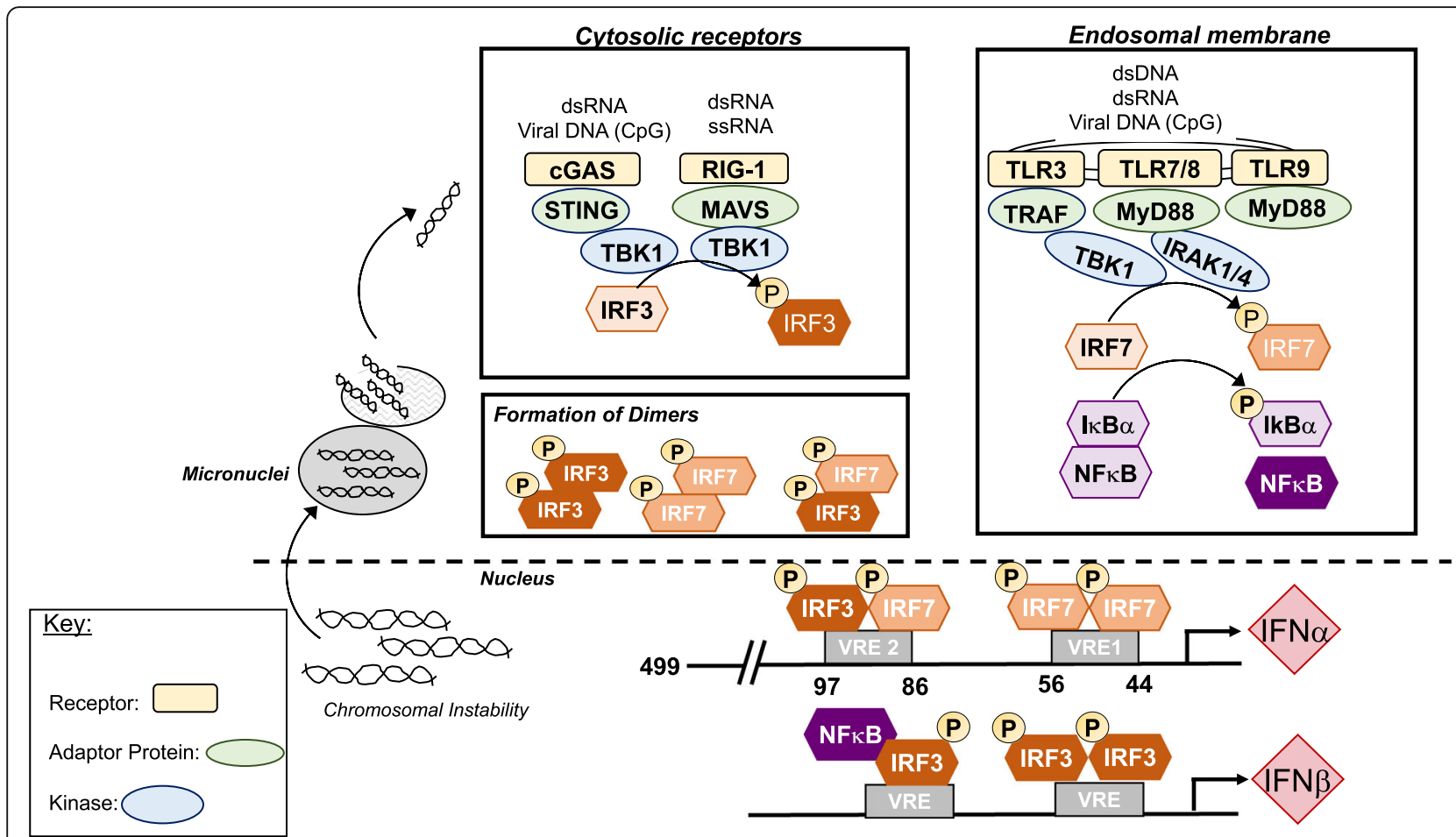

Fig. 2 Transcriptional activation of interferon alpha and beta: Type 1 interferon signaling may be stimulated by either cytosolic receptors or endosomal membrane receptors in the presence of double-stranded RNA (dsRNA), viral DNA, single-stranded RNA (ssRNA), or double-stranded DNA (dsDNA). Specific adaptor proteins bridge the receptor and the respective kinase. Upon kinase activation, IRF3 and IRF7 are phosphorylated by specific kinases and NFKB can be released from its inhibitory complex and translocate into the nucleus. Once IRF3 and IRF7 are phosphorylated, they form dimers. Dimerization partners are determined based on the site of phosphorylation and the levels of each protein. Once dimers form, they translocate into the nucleus and bind to the respective responsive element. IFNa has two viral response elements $30 \mathrm{~kb}$ apart (VRE1 and VRE2). VRE1 has preferential for IRF7 dimers whereas VRE2 has preferential binding for IRF3/RF7 dimers but both are not necessary for full transcription [26]. IFN $\beta$ is preferentially transcribed by IRF3 and NFkB. IFN $\beta$ can further promote the activation of IFNa through stimulating the IFNAR receptor which produces increased levels of IRF7 (Fig. 1)

chromosome segregation during mitosis thus leading to CIN which results in ruptured micronuclei releasing genomic DNA into the cytosol triggering an interferon response through sensing by cGAS-STING and subsequent phosphorylation of IRF3 [27, 28] (Fig. 2). A study analyzed CIN in a microarray dataset of lymph nodenegative primary breast cancer patients prior to systemic therapy and found that ER-negative, triplenegative, and basal-like tumors have significantly higher CIN scores [29] which is correlated with tumor metastasis [27]. Further supporting this data, a DNA microarray showed that compared to non-IBC samples, IBC had higher levels of TLRs, increasing the sensitivity of DNA damage response detection [5]. Therefore, IBC may be prone to an IFN $\alpha$ gene signature due to high levels of CIN but this remains to be tested.

Though there is limited information regarding IFN $\alpha$ in IBC, it is widely accepted that $\mathrm{NFK}_{\mathrm{K}} \mathrm{B}$ is elevated in IBC cell lines and patient tumors. IFN $\alpha$ could perhaps be controlled via NFKB indirectly since NFKB transcribes IFN $\beta$ which can promote transcription of IRF7 and IRF3, key regulators of IFNa [30]. Interestingly, it has been observed that the CD95/FADD apoptotic pathway regulates type I interferon production through stimulation of the $\mathrm{NFKB}$ axis thus leading to stemness, an intrinsic phenotype of IBC [31]. Stemness combines the ability of a cell to perpetuate its lineage, to give rise to differentiated cells and to interact with its environment to maintain a balance between quiescence, proliferation, and regeneration. Cancer stem cells (CSCs) display stemness in various circumstances, including the sustaining of cancer progression and the interaction with their environment in search for key survival factors. As a result, CSCs can recurrently persist after therapy which is probably a major contributing factor to the high rates of relapse observed for IBC.

Moving forward, it is important to consider investigating the genetic amplification of factors regulating IFN $\alpha$ signaling, post transcriptional modifications of these factors, and the intricate web of feedback and feedforward mechanisms that may be contributing to increased IFN $\alpha$. 


\section{Interferon alpha-stimulated genes in IBC tumors and resulting phenotypes}

We have established that IFN $\alpha$ may contribute to IBC tumor aggression; however, the specific role of IFN $\alpha$ is determined by many factors including its concentration, the specific tumor type, and the duration of the stimulus. For example, induction of antiviral activity requires a few hours of IFN $\alpha$ exposure whereas antiproliferative activity requires constant exposure or high concentrations of IFN $\alpha$ [32]. IFN $\alpha$ has been used as a potential therapeutic agent due to its ability to induce potent antiproliferative and even apoptotic cellular responses used at high concentrations, but concentrations of IFN $\alpha$ necessary for induction of apoptosis are a thousandfold higher than normally produced IFNa [32]. Therefore, ISGs produced at lower concentrations could be detrimental to the host by contributing to IBC cell tumorigenicity. IFN $\alpha$ can control proliferation, differentiation, survival, invasion, drug resistance, and radioresistance in tumors due to JAK/STAT activation and ISG expression [33]. Notably, these phenotypes may be present if JAK/ STAT signaling is not fully active.

\section{Non-canonical interferon stimulated gene induction by interferon alpha in IBC}

STATs can promote their effect independent of phosphorylation from canonical IFN $\alpha$ signaling as outlined in Fig. 1b. Canonically, during an interferon response, STATs are initially phosphorylated and IFN $\alpha$ signaling is initiated and resolved quickly, but during chronic inflammation STATs lose their phosphorylation status even in the presence of IFN $\alpha$ contributing to prolonged antiviral effects [21]. These stats are known as unphosphorylated STATs (U-STATS). A U-STAT1/U-STAT2 dimer with IRF9 forms an active, unphosphorylated ISGF3 (U-ISGF3) complex (Fig. 1b). In the presence of IFN, high levels of IRF9 may switch the balance from ISGF3-mediated transcription to U-ISGF3 signaling [34]. Importantly, ISGs stimulated by ISGF3 and by U-ISGF3 differ (Table 1), and we have found that TN-IBC cells lack basal levels of phosphorylated STAT1 and STAT2 in culture [14]. Aside from U-ISGF3 signaling, an ISGF3like complex can form between STAT2 and IRF9 which can transcribe ISGs independently of STAT1 [19]. However, in these same cells, treatment with a low dose of exogenous
IFN $\alpha$ promotes initial phospho-STAT activity but these levels decrease overtime while the expression of an ISG termed interferon-induced transmembrane protein-1 (IFITM1) production is markedly increased in TN-IBC cells [14]. This suggests that the IFNa pathway is not saturated at basal levels in TN-IBC indicating three things: (1) TN-IBC may still be able to respond to exogenous IFN treatment in both a pro- and anti-tumor manner depending on concentration; (2) this pathway may be stimulated on a basal level to confer autocrine signaling to the tumor, promoting increased aggression by mediating the levels of STAT1, STAT2, and IRF9; or (3) TN-IBC cells may not be sensitive to autocrine IFN $\alpha$ signaling. Therefore, depending on the quantity and the length of IFNa stimulus, different ISGs may be transcribed resulting in cellular phenotypic differences which may be specific to IBC as a whole or within IBC subtypes.

Aside from non-canonical IFN $\alpha$ signaling, IFN $\alpha$ is known to mediate activation of other cellular pathways through intracellular crosstalk. Interestingly, a mechanism whereby IFN $\alpha$ may lead to cellular aggression instead of apoptosis is through activation of $\mathrm{NFK} \mathrm{B}$ signaling via STAT3 and PI3K/AKT [35]. This pathway is independent of the ISRE-driven ISG gene expression, and not all ISGs have NFKB binding sites. Future studies elucidating these relationships may provide insight into how to target TN-IBC since NFkB has been shown to be elevated in IBC. Previous studies investigating autocrine IFN $\alpha$ signaling crosstalk are sparse and focus mainly on cellular responses to exogenous IFNo treatment. Studies have suggested IFN $\alpha$ can modulate PDGF and TGF $\beta$ signaling which could provide insight into autocrine IFN $\alpha$ signaling under the caveat that cellular responses to IFN are dose and time dependent [36]. The plasticity of the cellular response to IFN $\alpha$ has been extensively reviewed by Cheon et al. [37] and Medrano et al. [32].

Emerging evidence suggests that ISGs are responsible for the aggressive phenotype of many cancers. A few of which are outlined in Table 2 and how they are implicated in IBC [14, 17, 22, 38]. An emerging player in TN-IBC is IFITM1. IFITM1 is a $17-\mathrm{kDa}$ transmembrane protein located on $11 \mathrm{p} 15$ and can be transcribed in all cell types. IFITM1 is well known as a mediator of viral entry and replication and has recently gained popularity

Table 2 Interferon-stimulated genes implicated in IBC and their specific functions

\begin{tabular}{llll}
\hline Function & Function in IBC & ISG & Reference \\
\hline DNA damage resistance & Inferred & STAT1, IFI27, MX1/2, PLSCR1, OAS1/2/3, IRF9, IFITM1 & [14, 38] \\
Migration/invasion & Confirmed & IFITM1, STAT2 & [14] \\
Positive feedback & Inferred & IRF3, IRF7, IRF9 & [22] \\
Negative regulation & Inferred & SOCS1, USP18 & [17, 22] \\
\hline
\end{tabular}

A selected list of ISGs stimulated by IFNa is listed with corresponding function derived from the literature. The confirmation of the function of ISGs in IBC is outlined. All ISGs have confirmed expression in IBC in our unpublished data 
in cancer. IFITM1 may be induced by ISGF3 or U-ISGF3 making this a non-canonical ISG. We have previously identified IFITM1 as an ISG overexpressed in TN-IBC compared to HER2+ IBC [14]. Inhibiting IFITM1 expression in TN-IBC attenuates the proliferation and migration of these cells [14]. Other studies in aromatase inhibitor-resistant breast cancer [39],colorectal cancer [40], gliomas, [41], and head and neck cancer [42] have reported similar function of IFITM1. Furthermore, IFITM1 has been implicated in the radioresistant phenotype in head and neck, breast, prostate, lung, and brain cancers as discussed by Weichselbaum et al. [38].

Though STATs are not active, low levels of IFNa may be sustaining ISG production. Perhaps this is due to IFN control on STATs themselves. We have previously shown that siRNA against IFN $\alpha$ decreases STAT2 levels but not STAT1 levels in TN-IBC. Additionally, loss of STAT2, but not STAT1, was associated with decreased proliferation and migration in TN-IBC cells [14]. Therefore, stabilization of the U-ISGF3 complex could be a potential mechanism whereby TN-IBC utilizes IFNa for aggression. Future studies are needed to determine if and what ISGs contribute a feedback mechanism for IFN $\alpha$ as well as how these signaling mechanisms are altered in the presence of exogenous IFN $\alpha$.

\section{Interferon alpha in the IBC tumor microenvironment}

IFN $\alpha$ production from the tumor cells may have the capability of educating the microenvironment to be pro-survival for tumor cells. Likewise, tumor microenvironment (TME) components may be able to promote or enhance the migratory and invasive capacities of IBC cells, perhaps through influencing JAK/STAT signaling. Interestingly, one study has shown that $63.3 \%$ of breast cancers are positive for IFN $\alpha$ and that stage III breast cancers had no IFNa binding to the receptor complex suggesting constitutive secretion of IFN $\alpha$ but a lack of autocrine signaling [43]. JAK/STAT signaling is known to be both a cell intrinsic and cell extrinsic mechanism of IBC aggression [8], but the molecular mechanisms of this reciprocal signaling remain to be elucidated. If this mechanism is understood, perhaps a therapy targeting both the tumor microenvironment and tumor cells can be developed.

In this section, we discuss the role of IFN $\alpha$ on immunosurveillance and how this may lead to neoplastic initiation and IBC progression followed by a discussion of how IFN $\alpha$ may impact different cell types within the IBC TME. Figure 3a outlines how initial immunosurveillance driven by IFN $\alpha$ may promote a cytotoxic response against the tumor, and Fig. 3b shows how IFN $\alpha$ from the tumor cell may promote an altered, pro-tumorigenic immune milieu specific to IBC.

\section{The contribution of interferon alpha to tumor cell immune} escape: current mechanistic hypotheses

As previously discussed, IBC tumor emboli consist of tumor cells and immune cells and are highly metastatic $[2,8]$. Therefore, we hypothesize that IFN $\alpha$ signaling may support the survival of cancer cells and their escape from detection in immune cells.

During initial tumor formation, immune cells surrounding the tumor will recognize tumor-specific antigens to eliminate the transformed cells. Canonically, IFN $\alpha$ is a known tumor suppressor by inducing cellular senescence and death through recruiting dendritic cells and activating $\mathrm{T}$ cells [44]. However, a review published by Coppe et al. [45] introduces the senescence-associated secretory phenotype (SASP) that may be altered due to chronic inflammation. Normal cells undergoing the DNA damage response increase secretion of inflammatory cytokines which may halt their division. During senescence, normal epithelial cells become transformed through destabilization of chromatin, increased DNA damage, or through mutations necessary for survival. The secretory phenotype of transformed cells then promotes a pro-tumorigenic microenvironment through immune suppression; therefore, production and release of IFN $\alpha$ may contribute to immune suppression by upregulating the levels of PD-L1 on the tumor cell [46] (Fig. 3b).

The mechanism by which immune and stromal cells may drive the upregulation of tumor secreted IFN $\alpha$ remains elusive. Perhaps the stroma releases paracrine signals to neoplastic cells which then maintain these signals through endogenous autocrine signaling, promoting transformation and allowing cancer to progress with an ever-changing microenvironment. Regarding this crosstalk, we hypothesize that low levels of IFN $\alpha$ produced from immune cells in the microenvironment promote the transformation of epithelial cells with an increase in IFN $\alpha$ which subsequently makes the microenvironment conducive to tumor progression. This has been observed through chronic treatment with IFN $\beta$, showing that specific ISGs remain activated in the absence of IFN $\beta$ as well as during chronic interferon signaling [21]. Moving forward, we will discuss how dendritic cells, tumor-associated macrophages, cancer-associated fibroblasts, and endothelial cells may contribute and respond to elevated IFN $\alpha$ from IBC tumor cells, promoting a protumorigenic TME milieu. For more information refer to Cheon et al. [37] and Medrano et al. [32] for a comprehensive review of the TME and type- 1 interferons.

\section{Dendritic cells}

Dendritic cells (DCs) are activated by and are key producers of IFN $\alpha$ [47]. There are two subgroups of dendritic cells, plasmacytoid-derived DCs (pDCs), and myeloid-derived $\mathrm{DCs}(\mathrm{mDCs})$; both are known for transformed cell elimination [48]. In normal DC activation by IFN $\alpha$, DCs 


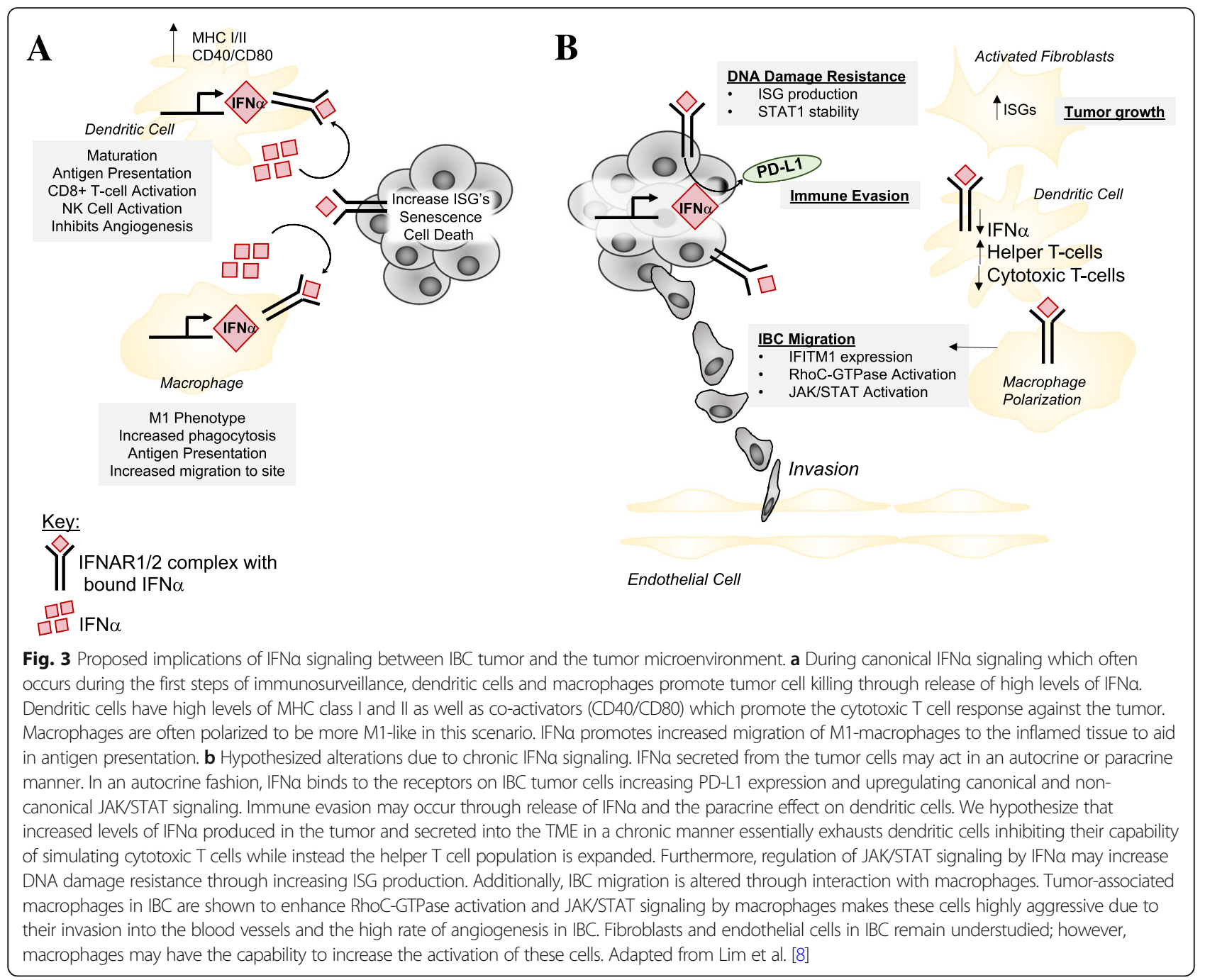

increase their levels of MHC class I and II, as well as T cell co-activators (CD40, CD80, CD86 and CD83) thus promoting a cytotoxic $\mathrm{T}$ cell response (Fig. 3a) [48]. Contrary to their canonical role, accumulation of mature dendritic cells has been correlated with increased metastasis in breast cancer [49]. Mature DCs are IFN $\alpha$ deficient thus promoting expansion of regulatory $\mathrm{T}$ cells associated with immune tolerance and poor clinical outcome [50]. This is in line with the current knowledge about DCs in IBC such that activated DCs are decreased in IBC [8]. Therefore, IFN $\alpha$ is an important mediator of dendritic cell activation and subsequent $\mathrm{T}$ cell response in the tumor microenvironment. We speculate that IBC secretion of IFN $\alpha$ essentially exhausts dendritic cells and $\mathrm{T}$ cells, therefore inhibiting the cytotoxic response (Fig. 3b).

\section{Tumor-associated macrophages}

Macrophages are the most abundant leukocyte present in the tumor stroma and display a high level of plasticity allowing adaptation to environmental stimuli. Despite popular evidence, macrophages do not exist as strictly M1 (anti-tumor) or M2 (pro-tumor) phenotypes, but instead lie within the spectrum. M1 polarization occurs due to exposure to interferon (IFN) $\gamma$, lipopolysaccharides (LPS), or TNF $\alpha$ produced by Th1, natural killer, or antigen presenting cells [51]. M1-polarized macrophages facilitate tumoricidal responses and inflammation [52]. M2 polarization is stimulated by IL-4, IL-13, and IL-10, or stress hormones, have poor antigen presenting capability, suppress T cell activation, and only exhibit minor cytotoxicity for tumor cells due to their limited ability to produce nitric oxide and pro-inflammatory cytokines [52].

IFN $\alpha$ is known to promote macrophages to favor the M1 phenotype (Fig. 3a) [53]; however, clinical and pre-clinical evidence in aggressive breast tumors and IBC seems to contradict this idea. Allaoui et al. [54] compared luminal, ER+ non-IBC, tumors and triplenegative non-IBC breast cancer tumors and found that the level of M2-like macrophages is significantly increased in triple-negative tumors. An in vitro study 
with SUM149 cells and THP-1 monocytes showed that during co-culture conditions; SUM149 cells promoted the immune suppressive phenotype of THP-1 monocytes [55]. Macrophages have further been shown to promote migration via RhoC-GTPase signaling in IBC (Fig. 3b) [11].

Perhaps IFN $\alpha$ secreted from the IBC tumor cells at a low dose avoids the rapid induction of M1 polarization while the chronic production eventually alters the macrophage phenotype, increasing the complexity of this relationship. Evidence suggests that macrophages are a key player in the microenvironment in IBC, yet many questions remain to be addressed. Specifically, how is IFN $\alpha$ a key regulator of macrophages within the IBC tumor TME and what are the other macrophage cell-cell relationships that are altered.

\section{Cancer-associated fibroblasts}

Cancer-associated fibroblasts (CAF's) are stromal cells that synthesize ECM and collagen and aid in wound healing. Though the involvement of CAFs in IBC is understudied, it is known that CAFs with high levels of IFN $\alpha$ response genes promote the growth of breast cancer MCF-7 (ER+, non-IBC) cells in an in vitro co-culture method (Fig. 3b) [56]. Though signaling processes are different between ER+ non-IBC and IBC breast cancer cells, this data provides insight into the importance of CAFs in breast cancer progression. Moreover, it has been shown that in aggressive breast cancer CAF levels are increased and that these activated CAFs can in turn regulate recruitment of macrophages [54]. Though IFN $\alpha$ may not have any known effects on CAFs in IBC, it can be postulated that the interplay between immune and stromal cells on the basis of the IFN $\alpha$ axis promotes IBC tumor aggression. Therefore, it is necessary to understand the effect of IFNa from IBC cells on the role of CAFs both in vitro and in vivo.

\section{Endothelial cells}

It is known that type I interferons normally have anti-angiogenic properties [57]. However, complex crosstalk between immune cells and stromal cells regulate tumor vasculature [32, 58]. IBC has elevated rates of infiltrating lymphatic endothelial cells compared to non-IBC [12]. Though this is regulated by VEGF signaling, the specific biological mechanisms promoting this are unknown. Perhaps the tumor cells do not directly act on the endothelial cells via IFN $\alpha$ signaling, but instead the interplay between the other immune and stromal cells previously discussed promotes increased infiltration. The direct relationship between IFN $\alpha, I B C$, and endothelial cells remains to be determined.

\section{Conclusion}

Evidence presented above suggests that IFN $\alpha$ is an important factor in IBC tumor development and progression but the functions of IFN $\alpha$ and how this cytokine may relate to other cellular processes remain unclear. IFNa signaling is comprised of multiple feedback loops, alternative signaling mechanisms, and communication between nearby cells contributing to multiple layers of system complexity. In IBC specifically, emerging evidence suggests that the tumor microenvironment is a key driver of onset and aggression. All cells can respond to IFN $\alpha$ and the response is dependent on concentration and tissue type making IFN $\alpha$ a highly pleiotropic cytokine.

IFN $\alpha$ as therapy has been extensively studied; however, targeting tumor produced IFN $\alpha$ or downstream signaling is new territory. Currently, ruxolitinib, a JAK1 and JAK2 inhibitor, is being investigated in treatment of primary and metastatic triple-negative breast cancer [59]. Ruxolitinib was well tolerated and, as expected, decreased JAK-STAT target genes and pSTAT3 activity; however, the primary efficacy endpoint was not met. The authors introduced the idea of intratumoral heterogeneity contributing to resistance to ruxolitinib. Perhaps, this could be due to alternative signaling mechanisms as outlined in Fig. 1b. Supporting our discussion of the importance of the tumor microenvironment in influencing IFN $\alpha$ signaling, these authors provide evidence for altered immune profiles based on tumor pSTAT3 and JAK2 expression. In addition to ruxolitinib, PD-L1 targeting therapies have emerged as a promising target for solid tumors including IBC and it has been discussed that IFN $\alpha$ can modulate tumor expression of PD-L1 [37]. Ultimately, the knowledge obtained by continued investigation of the role of type I interferon signaling in IBC not only sheds light on the molecular underpinnings of the disease but also provides novel mechanisms of targeted therapy and drug resistance to be considered in the context of translational medicine.

In future efforts to uncover the mechanistic insight into how IBC cells utilize IFN $\alpha$ for tumor progression, we must consider both intracellular signaling crosstalk and the tumor microenvironment. Doing so will help define the complexity of this system in hopes of contributing to the development of more efficacious therapies targeting both the tumor cell and the surrounding microenvironment.

\section{Abbreviations}

AAF: IFNa-activated factor; AKT: Protein kinase B; CAF: Cancer-associated fibroblast; CGAS-STING: Cyclic GMP-AMP synthase-stimulator of interferon genes; CIN: Chromosomal instability; CSC: Cancer stem cell; DC: Dendritic cell; dsDNA: Double-stranded DNA; dsRNA: Double-stranded RNA; ER: Estrogen receptor; FADD: Fas-associated death domain; HER2: Human epidermal growth factor 2; IBC: Inflammatory breast cancer; IFITM1: Interferon-induced transmembrane protein-1; IFN: Interferon; IFNA: Interferon alpha (gene); IFNAR1: Interferon alpha receptor 1; IFNAR2: Interferon alpha receptor 2; 
IFNGR: Interferon gamma receptor; IFNa: Interferon alpha; IFNß: Interferon beta; IFNY: Interferon gamma; IL10: Interleukin-10; IL13: Interleukin-13; IL4: Interleukin-4; IRAK: Interleukin-1 receptor associated kinase; IRF: Interferon regulatory factor; ISG: Interferon-stimulated gene; ISG 15: Interferon-stimulated gene-15: ISGF3: Interferon-stimulated gene factor 3; IKB: NFKB inhibitor; JAK: Janus-activated kinase; LPS: Lipopolysaccharide; MAVS: Mitochondrial antiviral signaling protein; mDC: Myeloid-derived DC; MHC: Major histocompatibility complex; MYD88: Myeloid differentiation primary response gene; NFkB: Nuclear factor kappa B; pCR: Pathological complete response; pDC: Plasmacytoid-derived DC; PDGF: Platelet-derived growth factor; PR: Progesterone receptor; RIG: Retinoic acidinducible gene; shRNA: Short

hairpin RNA; siRNA: Short interfering RNA; SOCS: Suppressor of cytokine signaling; ssRNA: Single-stranded RNA; STAT1: Signal transducer and activator of transcription-1; STAT2: Signal transducer and activator of transcription-2; STING: Stimulator of interferon genes; TAM: Tumor-associated macrophage; TBK: TANK-binding kinase; TLR: Toll-like receptor; TME: Tumor microenvironment; TN: Triple-negative; TNFa: Tumor necrosis factor alpha; TN-IBC: Triple-negative inflammatory breast cancer; T-reg: Regulatory T cells, CD4+; TYK2: Tyrosine kinase 2; U-ISGF3: Unphosphorylated interferon-stimulated gene factor 3;

USP18: Ubiquitin-specific peptidase 18; VEGF: Vascular endothelial growth factor

\section{Acknowledgements}

None

\section{Funding}

This study was supported, in part, by grants from the Department of Defense (W81XWH-12-1-0139 to JLW), start-up funds from the University of Kansas Medical Center (KUMC) (to JLW and OKP), and the NCI Cancer Center Support Grant (P30 (A168524 to JLW)

\section{Availability of data and materials}

None

\section{Authors' contributions}

OKP performed the literature search, wrote and edited the manuscript, and generated the figures. JLW conceptualized the paper, wrote and edited the manuscript, and provided the overall supervision and coordination of manuscript preparation. All authors were involved in writing the manuscript and approved the submitted version.

\section{Ethics approval and consent to participate \\ None}

\section{Consent for publication}

None

\section{Competing interests}

The authors declare that they have no competing interests.

\section{Publisher's Note}

Springer Nature remains neutral with regard to jurisdictional claims in published maps and institutional affiliations.

\section{Published online: 06 May 2019}

\section{References}

1. Hance KW, Anderson WF, Devesa SS, Young HA, Levine PH. Trends in inflammatory breast carcinoma incidence and survival: the surveillance, epidemiology, and end results program at the National Cancer Institute. J Natl Cancer Inst. 2005;97(13):966-75.

2. Ueno NT, Espinosa Fernandez JR, Cristofanilli M, Overmoyer B, Rea D, Berdichevski F, et al. International consensus on the clinical management of inflammatory breast cancer from the Morgan Welch Inflammatory Breast Cancer Research Program 10th Anniversary Conference. J Cancer. 2018;9(8):1437-47.

3. Low JA, Berman AW, Steinberg SM, Danforth DN, Lippman ME, Swain SM. Long-term follow-up for locally advanced and inflammatory breast cancer patients treated with multimodality therapy. J Clin Oncol. 2004;22(20):4067-74.

4. Prat A, Perou CM. Deconstructing the molecular portraits of breast cancer. Mol Oncol. 2011;5(1):5-23.

5. Van Laere SJ, Ueno NT, Finetti P, Vermeulen P, Lucci A, Robertson FM, et al. Uncovering the molecular secrets of inflammatory breast cancer biology: an integrated analysis of three distinct affymetrix gene expression datasets. Clin Cancer Res. 2013;19(17):4685-96.

6. Morrow RJ, Etemadi N, Yeo B, Ernst M. Challenging a misnomer? The role of inflammatory pathways in inflammatory breast cancer. Mediat Inflamm. 2017;2017:4754827.

7. Masuda H, Baggerly KA, Wang Y, Iwamoto T, Brewer T, Pusztai L, et al. Comparison of molecular subtype distribution in triple-negative inflammatory and non-inflammatory breast cancers. Breast Cancer Res. 2013;15(6):R112.

8. Lim B, Woodward WA, Wang X, Reuben JM, Ueno NT. Inflammatory breast cancer biology: the tumour microenvironment is key. Nat Rev Cancer. 2018; 18:485-89.

9. Luo H, Tu G, Liu Z, Liu M. Cancer-associated fibroblasts: a multifaceted driver of breast cancer progression. Cancer Lett. 2015;361(2):155-63.

10. Mohamed MM, El-Ghonaimy EA, Nouh MA, Schneider RJ, Sloane BF, ElShinawi M. Cytokines secreted by macrophages isolated from tumor microenvironment of inflammatory breast cancer patients possess chemotactic properties. Int J Biochem Cell Biol. 2014:46:138-47.

11. Allen SG, Chen YC, Madden JM, Fournier CL, Altemus MA, Hiziroglu AB, et al. Macrophages enhance migration in inflammatory breast cancer cells via RhoC GTPase signaling. Sci Rep. 2016;6:39190.

12. Colpaert CG, Vermeulen PB, Benoy I, Soubry A, van Roy F, van Beest P, et al. Inflammatory breast cancer shows angiogenesis with high endothelial proliferation rate and strong E-cadherin expression. Br J Cancer. 2003;88(5):718-25.

13. Bertucci F, Finetti $P$, Vermeulen $P$, Van Dam P, Dirix L, Birnbaum D, et al. Genomic profiling of inflammatory breast cancer: a review. Breast. 2014 23(5):538-45.

14. Ogony J, Choi HJ, Lui A, Cristofanilli M, Lewis-Wambi J. Interferon-induced transmembrane protein 1 (IFITM1) overexpression enhances the aggressive phenotype of SUM149 inflammatory breast cancer cells in a signal transducer and activator of transcription 2 (STAT2)-dependent manner. Breast Cancer Res. 2016;18(1):25.

15. Theofilopoulos AN, Baccala R, Beutler B, Kono DH. Type I interferons (alpha/ beta) in immunity and autoimmunity. Annu Rev Immunol. 2005;23:307-36.

16. Buess $M$, Nuyten DS, Hastie $T$, Nielsen $T$, Pesich $R$, Brown PO. Characterization of heterotypic interaction effects in vitro to deconvolute global gene expression profiles in cancer. Genome Biol. 2007;8(9):R191.

17. Schreiber $G$. The molecular basis for differential type I interferon signaling. J Biol Chem. 2017;292(18):7285-94.

18. Bordens R, Grossberg SE, Trotta PP, Nagabbushan TL. Molecular and biologic charaterization of recombinant interferon-a2b. Semin Oncol. 1997; 24(9):41-51.

19. Blaszczyk K, Nowicka H, Kostyrko K, Antonczyk A, Wesoly J, Bluyssen HA. The unique role of STAT2 in constitutive and IFN-induced transcription and antiviral responses. Cytokine Growth Factor Rev. 2016;29:71-81.

20. Stark GR, Kerr IM, Williams BR, Silverman RH, Schreiber RD. How cells respond to interferons. Annu Rev Biochem. 1998;67:227-64

21. Cheon H, Holvey-Bates EG, Schoggins JW, Forster S, Hertzog P, Imanaka N, et al. IFNbeta-dependent increases in STAT1, STAT2, and IRF9 mediate resistance to viruses and DNA damage. EMBO J. 2013:32(20):2751-63.

22. Schneider WM, Chevillotte MD, Rice CM. Interferon-stimulated genes: a complex web of host defenses. Annu Rev Immunol. 2014:32:513-45.

23. Platanias LC. Mechanisms of type-l- and type-II-interferon-mediated signalling. Nat Rev Immunol. 2005;5(5):375-86.

24. Yoneyama M, Kikuchi M, Natsukawa T, Shinobu N, Imaizumi T, Miyagishi M, et al. The RNA helicase RIG-I has an essential function in double-stranded RNA-induced innate antiviral responses. Nat Immunol. 2004;5(7):730-7.

25. Kawasaki T, Kawai T. Toll-like receptor signaling pathways. Front Immunol. 2014:5:461

26. Genin P, Vaccaro A, Civas A. The role of differential expression of human interferon--a genes in antiviral immunity. Cytokine Growth Factor Rev. 2009; 20(4):283-95.

27. Bakhoum SF, Ngo B, Laughney AM, Cavallo JA, Murphy CJ, Ly P, et al. Chromosomal instability drives metastasis through a cytosolic DNA response. Nature. 2018:553(7689):467-72.

28. Hartlova A, Erttmann SF, Raffi FA, Schmalz AM, Resch U, Anugula S, et al. DNA damage primes the type I interferon system via the cytosolic DNA sensor STING to promote anti-microbial innate immunity. Immunity. 2015; 42(2):332-43.

29. Smid M, Hoes $M$, Sieuwerts AM, Sleijfer S, Zhang $Y$, Wang $Y$, et al. Patterns and incidence of chromosomal instability and their prognostic relevance in breast cancer subtypes. Breast Cancer Res Treat. 2011;128(1):23-30. 
30. Panne D, Maniatis T, Harrison SC. An atomic model of the interferon-beta enhanceosome. Cell. 2007;129(6):1111-23.

31. Qadir AS, Ceppi P, Brockway S, Law C, Mu L, Khodarev NN, et al. CD95/Fas increases stemness in cancer cells by inducing a STAT1-dependent type I interferon response. Cell Rep. 2017;18(10):2373-86.

32. Medrano R, Hunger A, Mendonca S, Barbuto J, Strauss B. Immunomodulatory and antitumor effects of type I interferons and their application in cancer therapy. Oncotarget. 2017;8(41):71249-84.

33. Khodarev NN, Roizman B, Weichselbaum RR. Molecular pathways: interferon/stat1 pathway: role in the tumor resistance to genotoxic stress and aggressive growth. Clin Cancer Res. 2012;18(11):3015-21.

34. Sung PS, Cheon $\mathrm{H}$, Cho $\mathrm{CH}$, Hong SH, Park DY, Seo Hl, et al. Roles of unphosphorylated ISGF3 in HCV infection and interferon responsiveness. Proc Natl Acad Sci U S A. 2015;112(33):10443-8.

35. Pfeffer $L M$. The role of nuclear factor kappaB in the interferon response. J Interf Cytokine Res. 2011;31(7):553-9.

36. Wickenhauser C, Schmitz B, Selbach B, Brockbals C, Manske O, Thiele J. Interferon alpha2b directly induces fibroblast proliferation and transforming growth factor beta secretion of macrophages. $\mathrm{Br} J$ Haematol. 2000;109(2):296-304.

37. Cheon $\mathrm{H}$, Borden EC, Stark GR. Interferons and their stimulated genes in the tumor microenvironment. Semin Oncol. 2014;41(2):156-73.

38. Weichselbaum RR, Ishwaran H, Yoon T, Nuyten DS, Baker SW, Khodarev N, et al. An interferon-related gene signature for DNA damage resistance is a predictive marker for chemotherapy and radiation for breast cancer. Proc Natl Acad Sci U S A. 2008;105(47):18490-5.

39. Lui AJ, Geanes ES, Ogony J, Behbod F, Marquess J, Valdez K, et al. IFITM1 suppression blocks proliferation and invasion of aromatase inhibitorresistant breast cancer in vivo by JAK/STAT-mediated induction of p21. Cancer Lett. 2017;399:29-43.

40. Yu F, Xie D, Ng SS, Lum CT, Cai MY, Cheung WK, et al. IFITM1 promotes the metastasis of human colorectal cancer via CAV-1. Cancer Lett. 2015;368(1): 135-43.

41. Yu F, Ng SS, Chow BK, Sze J, Lu G, Poon WS, et al. Knockdown of interferoninduced transmembrane protein 1 (IFITM1) inhibits proliferation, migration, and invasion of glioma cells. J Neuro-Oncol. 2011;103(2):187-95.

42. Hatano H, Kudo Y, Ogawa I, Tsunematsu T, Kikuchi A, Abiko Y, et al. IFNinduced transmembrane protein 1 promotes invasion at early stage of head and neck cancer progression. Clin Cancer Res. 2008;14(19):6097-105.

43. Ahmed F, Mahmood N, Shahid S, Hussain Z, Ahmed I, Jalal A, et al. Mutations in human interferon alpha2b gene and potential as risk factor associated with female breast cancer. Cancer Biother Radiopharm. 2016; 31(6):199-208

44. Leplina OY, Tyrinova TV, Tikhonova MA, Ostanin AA, Chernykh ER. Interferon alpha induces generation of semi-mature dendritic cells with high proinflammatory and cytotoxic potential. Cytokine. 2015;71(1):1-7.

45. Coppe JP, Desprez PY, Krtolica A, Campisi J. The senescence-associated secretory phenotype: the dark side of tumor suppression. Annu Rev Pathol. 2010:5:99-118.

46. Huang B, Zhao J, Li H, He KL, Chen Y, Chen SH, et al. Toll-like receptors on tumor cells facilitate evasion of immune surveillance. Cancer Res. 2005;65(12):5009-14.

47. Gardner A, Ruffell B. Dendritic cells and cancer immunity. Trends Immunol. 2016;37(12):855-65.

48. Palucka K, Coussens LM, O'Shaughnessy J. Dendritic cells, inflammation, and breast cancer. Cancer J. 2013:19(6):511-6.

49. Treilleux I, Blay JY, Bendriss-Vermare N, Ray-Coquard I, Bachelot T, Guastalla $J P$, et al. Dendritic cell infiltration and prognosis of early stage breast cancer. Clin Cancer Res. 2004;10(22):7466-74.

50. Sisirak V, Faget J, Gobert M, Goutagny N, Vey N, Treilleux I, et al. Impaired IFN-alpha production by plasmacytoid dendritic cells favors regulatory T-cell expansion that may contribute to breast cancer progression. Cancer Res. 2012;72(20):5188-97.

51. Sousa S, Brion R, Lintunen M, Kronqvist P, Sandholm J, Monkkonen J, et al. Human breast cancer cells educate macrophages toward the M2 activation status. Breast Cancer Res. 2015;17:101.

52. Porta C, Subhra Kumar B, Larghi P, Rubino L, Mancino A, Sica A. Tumor promotion by tumor-associated macrophages. Adv Exp Med Biol. 2007; 604:67-86.

53. Zhuang PY, Shen J, Zhu XD, Tang ZY, Qin LX, Sun HC. Direct transformation of lung microenvironment by interferon-a treatment counteracts growth of lung metastasis of hepatocellular carcinoma. PLoS One. 2013;8(3):e58913.
54. Allaoui R, Bergenfelz C, Mohlin S, Hagerling C, Salari K, Werb Z, et al. Cancer-associated fibroblast-secreted CXCL16 attracts monocytes to promote stroma activation in triple-negative breast cancers. Nat Commun. 2016:7:13050.

55. Stewart DA, Yang Y, Makowski L, Troester MA. Basal-like breast cancer cells induce phenotypic and genomic changes in macrophages. Mol Cancer Res. 2012;10(6):727-38.

56. Hosein AN, Livingstone J, Buchanan M, Reid JF, Hallett M, Basik M. A functional in vitro model of heterotypic interactions reveals a role for interferon-positive carcinoma associated fibroblasts in breast cancer. BMC Cancer. 2015:15:130.

57. Indraccolo S. Interferon-alpha as angiogenesis inhibitor: learning from tumor models. Autoimmunity. 2010;43(3):244-7.

58. Norton KA, Jin K, Popel AS. Modeling triple-negative breast cancer heterogeneity: effects of stromal macrophages, fibroblasts and tumor vasculature. J Theor Biol. 2018;452:56-68.

59. Stover DG, Gil Del Alcazar CR, Brock J, Guo H, Overmoyer B, Balko J, et al. Phase II study of ruxolitinib, a selective JAK1/2 inhibitor, in patients with metastatic triple-negative breast cancer. NPJ Breast Cancer. 2018;4:10.

\section{Ready to submit your research? Choose BMC and benefit from:}

- fast, convenient online submission

- thorough peer review by experienced researchers in your field

- rapid publication on acceptance

- support for research data, including large and complex data types

- gold Open Access which fosters wider collaboration and increased citations

- maximum visibility for your research: over $100 \mathrm{M}$ website views per year

At BMC, research is always in progress.

Learn more biomedcentral.com/submissions 